City University of New York (CUNY)

CUNY Academic Works

Publications and Research

CUNY Graduate School of Public Health \&

Health Policy

2015

\title{
Maternal Characteristics and Clinical Diagnoses Influence Obstetrical Outcomes in Indonesia
}

Asri Adisasmita

University of Indonesia

Carl V. Smith

University of Nebraska at Omaha

Ayman A.E. El-Mohandes

CUNY School of Public Health

Poppy Elvira Deviany

University of Indonesia

Judith J. Ryon

John Snow, Inc.

See next page for additional authors

\section{How does access to this work benefit you? Let us know!}

More information about this work at: https://academicworks.cuny.edu/sph_pubs/206

Discover additional works at: https://academicworks.cuny.edu

This work is made publicly available by the City University of New York (CUNY).

Contact: AcademicWorks@cuny.edu 


\section{Authors}

Asri Adisasmita, Carl V. Smith, Ayman A.E. El-Mohandes, Poppy Elvira Deviany, Judith J. Ryon, Michele Kiely, Quail Rogers-Bloch, and Reginald F. Gipson 


\title{
Maternal Characteristics and Clinical Diagnoses Influence Obstetrical Outcomes in Indonesia
}

\author{
Asri Adisasmita $\cdot$ Carl V. Smith · Ayman A. E. El-Mohandes • \\ Poppy Elvira Deviany • Judith J. Ryon • Michele Kiely • \\ Quail Rogers-Bloch $\cdot$ Reginald F. Gipson
}

Published online: 6 February 2015

(c) Springer Science+Business Media New York 2015

\begin{abstract}
This Indonesian study evaluates associations between near-miss status/death with maternal demographic, health care characteristics, and obstetrical complications, comparing results using retrospective and prospective data. The main outcome measures were obstetric conditions and socio-economic factors to predict near-miss/death. We abstracted all obstetric admissions (1,358 retrospective and 1,240 prospective) from two district hospitals in East Java, Indonesia between 4/1/2009 and $5 / 15 / 2010$. Prospective data added socio-economic status, access to care and referral patterns. Reduced logistic
\end{abstract}

Judith J. Ryon was formerly at John Snow, Inc., Arlington, VA, USA.

A. Adisasmita

Department of Epidemiology, Faculty of Public Health,

University of Indonesia, Depok, West Java, Indonesia

C. V. Smith

College of Medicine, University of Nebraska Medical Center,

Omaha, NE, USA

A. A. E. El-Mohandes

School of Public Health, City University of New York,

New York, NY, USA

P. E. Deviany

Center for Family Welfare, University of Indonesia, Depok,

West Java, Indonesia

J. J. Ryon · Q. Rogers-Bloch

John Snow, Inc., Arlington, VA, USA

M. Kiely $(\square)$

School of Public Health, City University of New York, 555 West

57th St., Room 1135, New York, NY 10019, USA

e-mail: michele.kiely@sph.cuny.edu

R. F. Gipson

Health Improvement Project, Dili, Timor Leste models were constructed, and multivariate analyses used to assess association of risk variables to outcome. Using multivariate analysis, variables associated with risk of near-miss/death include postpartum hemorrhage (retrospective AOR 5.41, $95 \%$ CI 2.64-11.08; prospective AOR 10.45 , $95 \%$ CI 5.59-19.52) and severe preeclampsia/ eclampsia (retrospective AOR 1.94, $95 \%$ CI 1.05-3.57; prospective AOR 3.26, $95 \%$ CI 1.79-5.94). Associations with near-miss/death were seen for antepartum hemorrhage in retrospective data (AOR 9.34, $95 \%$ CI 4.34-20.13), and prospectively for poverty (AOR $2.17,95 \%$ CI 1.33-3.54) and delivering outside the hospital (AOR 2.04, $95 \%$ CI 1.08-3.82). Postpartum hemorrhage and severe preeclampsia/eclampsia are leading causes of near-miss/death in Indonesia. Poverty and delivery outside the hospital are significant risk factors. Prompt recognition of complications, timely referrals, standardized care protocols, prompt hospital triage, and structured provider education may reduce obstetric mortality and morbidity. Retrospective data were reliable, but prospective data provided valuable information about barriers to care and referral patterns.

Keywords Maternal near-miss · Pregnancy - Indonesia * Risk factors

\section{Introduction}

Worldwide approximately 289,000 women die annually from complications of pregnancy and childbirth. Many more experience morbidity and near-miss events. The vast majority of deaths and adverse outcomes occur in developing countries, where birth rates are the highest [1-3]. While approximately one third of maternal deaths occur in South Asia [2], Indonesia [4] as the world's fourth most 
populous country, contributes a large number of these deaths. The IDHS (2012) in Indonesia reports an increased MMR to 359/100,000 [5]. This statistic further removes Indonesia from it declared MDG goal of 102 maternal deaths per 100,000 live births in 2015. It is apparent that the MMR is higher in Indonesia than in other neighboring countries, for example, the Philippines $(99 / 100,000)$ and Vietnam $(59 / 100,000)[6]$.

As part of achieving the Millennium Development Goals, a recommended strategy was that all births be managed by skilled health professionals. In an effort to implement this initiative, Indonesia posted more than 54,000 midwives, doubling the proportion of births managed by a midwife or other health professional from $35 \%$ in the late 1980s to $69 \%$ in 2000 [7]. During this increase in management of birth by skilled health professionals, in East Java, only $17.2 \%$ of deliveries occurred in district and provincial hospitals, in contrast to $40 \%$ of deaths [8], with $49 \%$ of hospital deliveries classified as complicated [8-10]. Available evidence from elsewhere in Indonesia suggests that inadequate care may contribute to $60 \%$ of maternal hospital deaths [11]. Women who present to the hospital are the sickest, with the most complicated cases. These facilities are considered the final referral point for community providers of obstetrical care. Hospital services at the district level and above need to provide accessible, responsive and comprehensive care to these patients.

Indonesia has made substantial investments in primary care and community based services for mothers and neonates over the last two decades [12]. As part of this effort, Indonesia has trained midwives and developed standardized curricula. There is a need to understand the impact of maternal risk factors such as low educational attainment, poverty, and barriers/access to care on poor reproductive outcomes, with a goal of employing the most effective approaches to improving outcomes. Better elucidation of the clinical and socio-demographic risk profiles of women seeking hospital care may help the health care system prepare for its role in reducing maternal mortality in Indonesia.

A systematic review on the prevalence of near-miss events, based on disease criteria, ranged between 0.6 and $14.98 \%$ [13]. The ratio of mortality to near-miss found in several studies were 1:4.8 in Nigeria [14], 1:7 in Pakistan [15] and Kathmandu [16],1:8.6 in Brazil [17], 1:118 in the UK [18], and 1:185 in 8 Latin American countries [19]. Putting this in perspective, with approximately 289,000 maternal deaths in 2013 worldwide, the WHO considers Southeast Asia to have a moderate rate of maternal mortality. Of other countries in Southeast Asia, Timor-Leste and Myanmar are the only ones with higher MMR rates. Because of its population size, Indonesia contributes a much larger number of actual deaths [20].
Despite high rates of maternal mortality, death is a relatively rare event in the clinical care environment. Consequently, we chose to investigate near-miss events in addition to deaths. Near-miss events are strongly correlated with death and may provide relevant insight into the impact of maternal factors and quality of obstetric care on outcomes [21-23]. Our primary goal was to evaluate the associations between maternal demographic and health care related characteristics and obstetric diagnoses with mode of delivery and near-miss and death in two district hospitals in East Java. The study also compared results based on retrospective versus prospectively collected data.

\section{Methods}

The lead investigative team conducting this study is affiliated with the Center for Family Welfare at the School of Public Health at The University of Indonesia. The study was implemented at two district hospitals in East Java, Bangil and Kanjuruhan. The Institutional Review Boards of the university and both hospitals approved this protocol prior to implementation.

Bangil District Hospital is located in a coastal area of the Madura Straight. This hospital performs obstetric surgery and has the larger number of annual deliveries. This facility has a small number of private hospitals surrounding it, which provide obstetric services. Staff included nurses, midwives, obstetricians and pediatricians but the hospital does not have an intensive care unit (ICU). In contrast, the Kanjuruhan District hospital has an ICU, is surrounded by nine private hospitals that provide emergency obstetric services, and is located in a more mountainous region in the south-central part of the province. Staffing is similar to Bangil, but because of its ICU, it is more likely to receive referrals from other hospitals in the region.

Maternal near miss is defined as "a woman who nearly died but survived a complication that occurred during pregnancy, childbirth or within 42 days of termination of pregnancy" [24]. The precise criteria for this study were drawn from a previous study on the definition and frequency of obstetric near-miss events in West Java, Indonesia, based on a national consensus on the criteria for near-miss definition [25], that modified criteria proposed by Mantel and colleagues [26] (See Table 1). The mortality index measures the quality of emergency obstetric care for life-threatening conditions [27].

Poverty status was determined by a patient's possession of a letter from their village leader verifying poverty and therefore eligibility for government insurance for the poor. Failure to obtain such a letter of poverty is a substantial barrier to receiving care in Indonesia. 


\section{Participants}

We completed a retrospective abstraction of all medical records for obstetrical admissions between April 1, 2009 and September 30, $2009(\mathrm{~N}=1,358)$. We similarly recorded data prospectively on all admissions from October 1, 2009 through May 15, $2010(\mathrm{~N}=1,240)$ at the two delivery sites. Due to the relatively short time to conduct the study, the research team felt the number of women experiencing obstetric complications would be small. In order to maximize the number of observed complications, the team decided to include both retrospective and prospective data. Provided there were no salient differences in the diagnoses and the associated outcomes observed between the two data sets, the team decided that for future research, retrospective and prospectively collected data could be combined.

\section{Measures}

Data included age, parity, socioeconomic status, educational level, insurance status, final diagnosis, residence (urban/rural), mode of delivery, the presence of complications, birth outcomes and condition on discharge. The outcome of interest was presence of near-miss or maternal death. Data were obtained from medical records, registers of delivery ward, obstetric ward, operating theater, Kanjuruhan Hospital ICU, as well as nurse's daily treatment(s) book, notes on vital signs, and nurse's hand-over notes. The data from different sources were linked based on the woman's hospital admission number.

This study included all types of obstetric and nonobstetric complications, including deaths and near-miss events. Near-miss events were defined for women admitted during pregnancy, intra-partum or postpartum who survived a life-threatening condition based on the criteria defined by Adisasmita et al. [25].

Data on socio-economic status, access to care (including transportation and geographic difficulties), and referral patterns were obtained by interviewing the women or their family during hospitalization using a separate structured interview form. These interview data were available only for prospective cases. Payment methods were available for both groups and were used as an additional proxy for socioeconomic status and access to care. Mothers were classified into SES quintiles based on the methodology used in the Indonesia DHS report [28].

Three physicians at each hospital used structured data extraction forms to collect data. Prior to data collection, physicians participated in a 1-week intensive training to ensure data accuracy and consistency. Field supervisors conducted periodic checks to maintain the integrity of the

Table 1 Criteria for inclusion of near miss cases (modified from Mantel et al. 1998)

\begin{tabular}{|c|c|}
\hline \\
\hline \multicolumn{2}{|l|}{$\begin{array}{l}\text { Organ dysfunction } \\
\text { Cardiac dysfunction } \\
\text { Pulmonary embolism }\end{array}$} \\
\hline Vascular dysfunction & $\begin{array}{l}\text { Hypovolemic requiring two or more units of blood, blood loss with hypovolemic shock (systolic blood pressure } \\
<90 \mathrm{mmHg} \text { or undetectable pulse), infusion and/or transfusion of } \geq 11 \text { in } 2 \mathrm{~h} \text {, free flow infusion }{ }^{\mathrm{a}} \text {, massive } \\
\text { hemorrhage recorded in notes }\end{array}$ \\
\hline $\begin{array}{l}\text { Immunological } \\
\text { dysfunction }\end{array}$ & Septic shock \\
\hline $\begin{array}{l}\text { Respiratory } \\
\text { dysfunction }\end{array}$ & $\begin{array}{l}\text { Intubation or ventilation for reasons other than general anesthesia, oxygen saturation on pulse oximetry }<90 \% \text { leading } \\
\text { to ventilation }\end{array}$ \\
\hline Renal dysfunction & $\begin{array}{l}\text { Oliguria }<30 \mathrm{ml} \text { per hour or }<400 \mathrm{ml} \text { per } 24 \mathrm{~h} \text {, shock not responsive to intravascular rehydration or diuresis, } \\
\text { hemodialysis }\end{array}$ \\
\hline Liver dysfunction & $\begin{array}{l}\text { Jaundice in pre-eclampsia, abnormal liver function tests (Alanine aminotransferase (ALT) or SGPT } \geq 40 \mathrm{IU} / \mathrm{L} \text {, and/or } \\
\text { AST or SGOT } \geq 70 \mathrm{IU} / \mathrm{L} \text {, and/or LDH } \geq 600 \mathrm{IU} / \mathrm{L} \text {, and/or Bilirubin } \geq 1.2 \mathrm{mg} / \mathrm{dl} \text { ) in the presence of eclampsia or } \\
\text { preeclampsia or hypertension }\end{array}$ \\
\hline $\begin{array}{l}\text { Coagulation } \\
\text { dysfunction }\end{array}$ & $\begin{array}{l}\text { Acute thrombocytopenia, of }<100,000 / \mathrm{mm}^{3} \text { associated with bleeding manifestations (e.g. petechia, epistaxis, melena, } \\
\text { purpura, heaturia), or Acute thrombocytopenia of }<52,000 / \mathrm{mm}^{3} \text { associated with or without bleeding manifestations } \\
\text { (e.g. petechia, epistaxis, melena, purpura, hematuria), prolonged bleeding time and clotting time ( } \geq 7 \mathrm{~min} \text { ), abnormal } \\
\text { Activated Partial Thromboplastin Time (APTT) or Prothrombin Time (PT), coagulopathy, DIC (Disseminated } \\
\text { Intravascular Coagulation) }\end{array}$ \\
\hline
\end{tabular}

Cerebral dysfunction Coma, cerebral edema, seizures other than eclampsia

Management based criteria

Intensive care admission, emergency hysterectomy, needs resuscitation, anesthetic accident, referral to tertiary hospital

Clinical diagnosis

Eclampsia, uterine rupture, ectopic pregnancy

\footnotetext{
${ }^{a}$ Free flow infusion refers to a massive infusion of fluids in the case of shock
} 
data collection process. They examined the case notes and further verified the diagnosis listed in the forms, the presence or absence of maternal complications, urgency of cesarean section, determined the presence of specific nearmiss criteria, if and when there were a near-miss event, as well as any deaths. Women admitted in critical condition (based upon vital signs at admission) were distinguished from those whose life-threatening condition developed during hospitalization. This is referred to as near-miss upon admission and is considered as acuity of illness upon arrival at the hospital and is associated with delay in seeking care. Mothers who died were also identified through all sources of information available in the hospital, including morgue data.

\section{Statistical Analysis}

Retrospectively and prospectively collected data were compared to examine the influence of timing of data collection on the risk variables and outcomes of interest to the study. Mode of delivery and maternal outcomes were stratified by the principal maternal diagnoses. Prospective clinical data were linked and merged with the interview data based on the study number. The goal was to predict outcomes based on socio-economic status, personal bar$\operatorname{rier}(\mathrm{s})$ (delay in seeking care, refusal to receive care), access barrier(s) to care (transportation, geographical, funds) and referral patterns.

Univariate analyses were conducted using all predictor variables on the outcomes of interest, including mode of delivery and maternal near-miss and death. These comparisons were conducted separately for the retrospective and prospective data sets. Odds ratios and their $95 \%$ confidence intervals $(\mathrm{CI})$ were calculated using unconditional logistic regression. Statistical significance was assessed through the likelihood ratio test [29]. Reduced logistic models were constructed based on significance level in the bivariate results, and multivariate analyses were then used to assess association of risk variables with maternal outcome. Backwards elimination using likelihood ratio was employed to build the final model based on specified criteria of $p$ value of 0.25 for entering the model and $p$ value of 0.05 for removal from the model. If variables demonstrated collinearity, only one was included in the model. The variable chosen was the one with highest level of statistical significance. The magnitude of the

Table 2 Maternal characteristics by period of study

\begin{tabular}{|c|c|c|c|c|}
\hline Maternal characteristics & $\begin{array}{l}\text { Retrospective data } \\
\text { (April-Sept'09) } \\
\mathrm{N}=1,358\end{array}$ & $\begin{array}{l}\text { Prospective data } \\
\text { (Oct'09-March'10) } \\
\mathrm{N}=1,240\end{array}$ & $p$ value & $\begin{array}{l}\text { Total } \\
\text { (April'09-March'10) } \\
\mathrm{N}=2,598\end{array}$ \\
\hline Age & $\mathrm{n}=1,347$ & $\mathrm{n}=1,235$ & & $\mathrm{n}=2,582$ \\
\hline$<20$ & $116(8.6 \%)$ & $144(11.7 \%)$ & 0.012 & $260(10.1 \%)$ \\
\hline $20-35$ & $999(74.2 \%)$ & $858(69.5 \%)$ & & $1,857(71.9 \%)$ \\
\hline$>35$ & $232(17.2 \%)$ & $233(18.9 \%)$ & & $465(18 \%)$ \\
\hline Gravidity & $\mathrm{n}=1,261$ & $\mathrm{n}=1,122$ & & $\mathrm{n}=2,383$ \\
\hline 1 & $531(42.1)$ & $501(44.7 \%)$ & 0.407 & $1,032(43.3 \%)$ \\
\hline $2-3$ & $548(43.5)$ & $459(40.9 \%)$ & & $1,007(42.3 \%)$ \\
\hline $4+$ & $182(14.4)$ & $162(14.4 \%)$ & & $344(14.4 \%)$ \\
\hline Insurance & $\mathrm{n}=1,341$ & $\mathrm{n}=1,225$ & & $\mathrm{n}=2,566$ \\
\hline Insurance for the poor & $656(48.9)$ & $601(49.1 \%)$ & 0.997 & $1,257(49 \%)$ \\
\hline Other insurance (including free of charge) & $96(7.2)$ & $87(7.1 \%)$ & & $183(7.1 \%)$ \\
\hline None & $589(43.9)$ & $537(43.8 \%)$ & & $1,126(43.9 \%)$ \\
\hline Residence & $\mathrm{n}=1,358$ & $\mathrm{n}=1,240$ & & $\mathrm{n}=2,598$ \\
\hline Urban & $297(21.9)$ & $251(20.2 \%)$ & 0.310 & $548(21.1 \%)$ \\
\hline Rural & $1,061(78.1)$ & $989(79.8 \%)$ & & $2,050(78.9 \%)$ \\
\hline Referral cases & $\mathrm{n}=1,358$ & $\mathrm{n}=1,240$ & & $\mathrm{n}=2,598$ \\
\hline Referred (from lower level facility) & $908(66.9 \%)$ & $819(66.0 \%)$ & 0.660 & $1,727(66.5 \%)$ \\
\hline Not referred & $450(33.1 \%)$ & $421(34.0 \%)$ & & $871(33.5 \%)$ \\
\hline Time of admission & $\mathrm{n}=1,337$ & $\mathrm{n}=1,234$ & & $\mathrm{n}=2,571$ \\
\hline Weekdays 1 (07:01-14:00) & $561(42 \%)$ & $527(42.7 \%)$ & 0.235 & $1,088(42.3 \%)$ \\
\hline Weekdays 2 (14:01-21:00) & $307(23 \%)$ & $304(24.6 \%)$ & & $611(23.8 \%)$ \\
\hline Weekdays 3 (21:01-07:00) & $260(19.4 \%)$ & $244(19.8 \%)$ & & $504(19.6 \%)$ \\
\hline Weekend & $209(15.6 \%)$ & $159(12.9 \%)$ & & $368(14.3 \%)$ \\
\hline
\end{tabular}


Table 3 Maternal diagnoses by period of study

\begin{tabular}{|c|c|c|c|c|}
\hline Maternal diagnosis ${ }^{\mathrm{a}}$ & $\begin{array}{l}\text { Retrospective data } \\
(4 / 2009-9 / 2009) \\
\mathrm{N}=1,358(100 \%)\end{array}$ & $\begin{array}{l}\text { Prospective data } \\
(10 / 2009-3 / 2010) \\
\mathrm{N}=1,240(100 \%)\end{array}$ & $\begin{array}{l}\text { Total } \\
(4 / 2009-3 / 2010) \\
\mathrm{N}=2,598(100 \%)\end{array}$ & $p$ value \\
\hline Normal (no maternal complication) & $128(9.4 \%)$ & $109(8.8 \%)$ & $237(9.1 \%)$ & 0.574 \\
\hline Antepartum hemorrhage & $85(6.3 \%)$ & $58(4.7 \%)$ & $143(5.5 \%)$ & 0.077 \\
\hline Postpartum hemorrhage & $91(6.7 \%)$ & $100(8.1 \%)$ & $191(7.4 \%)$ & 0.183 \\
\hline Severe preeclampsia/eclampsia & $194(14.3 \%)$ & $156(12.6 \%)$ & $350(13.5 \%)$ & 0.204 \\
\hline Miscarriage & $181(13.3 \%)$ & $177(14.3 \%)$ & $358(13.8 \%)$ & 0.485 \\
\hline Dystocia & $249(18.3 \%)$ & $214(17.3 \%)$ & $463(17.8 \%)$ & 0.473 \\
\hline Malpresentation & $137(10.1 \%)$ & $110(8.9 \%)$ & $247(9.5 \%)$ & 0.291 \\
\hline Time of near-miss occurrence & & & & 0.294 \\
\hline Near-miss on admission & $78(5.7 \%)$ & $85(6.9 \%)$ & $163(6.3 \%)$ & \\
\hline Near-miss after admission & $53(3.9 \%)$ & $62(5.0 \%)$ & $115(4.4 \%)$ & \\
\hline Near-miss undefined time & $35(2.6 \%)$ & $27(2.2 \%)$ & $62(2.4 \%)$ & \\
\hline Non-near-miss & $1,192(87.8 \%)$ & $1,066(86.0 \%)$ & $2,258(86.9 \%)$ & \\
\hline
\end{tabular}

Table 4 Mode of delivery of women delivered in the hospital by diagnoses

\begin{tabular}{|c|c|c|c|c|c|c|c|}
\hline \multicolumn{4}{|l|}{ Retrospective $(\mathrm{N}=916)$} & \multicolumn{4}{|l|}{ Prospective $(\mathrm{N}=792)$} \\
\hline \multirow[t]{2}{*}{ Diagnoses $^{\mathrm{a}}$} & \multicolumn{2}{|c|}{ Mode of delivery } & \multirow[t]{2}{*}{$\overline{p \text { value }}$} & \multirow[t]{2}{*}{ Diagnoses } & \multicolumn{2}{|c|}{ Mode of delivery } & \multirow[t]{2}{*}{$p$ value } \\
\hline & $\begin{array}{l}\text { C-section } \\
45 \% \\
\quad(n=414)\end{array}$ & $\begin{array}{l}\text { Vaginal } \\
\text { delivery } \\
55 \% \\
\quad(\mathrm{n}=502)\end{array}$ & & & $\begin{array}{l}\text { C-section } \\
42 \% \\
\quad(n=330)\end{array}$ & $\begin{array}{l}\text { Vaginal } \\
\text { delivery } \\
58 \% \\
\quad(\mathrm{n}=462)\end{array}$ & \\
\hline $\begin{array}{l}\text { Antepartum hemorrhage } \\
\quad(\mathrm{n}=54)\end{array}$ & $\begin{array}{l}83.3 \% \\
\quad(\mathrm{n}=45)\end{array}$ & $\begin{array}{l}16.7 \% \\
\quad(\mathrm{n}=9)\end{array}$ & $<0.001$ & $\begin{array}{l}\text { Antepartum hemorrhage } \\
\quad(\mathrm{n}=37)\end{array}$ & $\begin{array}{l}48.2 \% \\
\quad(\mathrm{n}=54)\end{array}$ & $\begin{array}{l}51.8 \% \\
\quad(\mathrm{n}=58)\end{array}$ & $<0.001$ \\
\hline $\begin{array}{l}\text { Severe pre/eclampsia } \\
\quad(\mathrm{n}=151)\end{array}$ & $\begin{array}{l}48.3 \% \\
\quad(\mathrm{n}=73)\end{array}$ & $\begin{array}{l}51.7 \% \\
\quad(\mathrm{n}=78)\end{array}$ & 0.395 & $\begin{array}{l}\text { Severe pre/eclampsia } \\
\quad(\mathrm{n}=112)\end{array}$ & $\begin{array}{l}48.2 \% \\
\quad(\mathrm{n}=54)\end{array}$ & $\begin{array}{l}51.8 \% \\
\quad(\mathrm{n}=58)\end{array}$ & 0.129 \\
\hline $\begin{array}{l}\text { Dystocia } \\
(\mathrm{n}=238)\end{array}$ & $\begin{array}{l}65.5 \% \\
\quad(\mathrm{n}=156)\end{array}$ & $\begin{array}{l}34.5 \% \\
\quad(\mathrm{n}=82)\end{array}$ & $<0.001$ & Dystocia $(\mathrm{n}=197)$ & $\begin{array}{l}62.9 \% \\
\quad(n=124)\end{array}$ & $\begin{array}{l}37.1 \% \\
\quad(\mathrm{n}=73)\end{array}$ & $<0.001$ \\
\hline $\begin{array}{l}\text { Malpresentation } \\
(\mathrm{n}=116)\end{array}$ & $\begin{array}{l}57.8 \% \\
\quad(\mathrm{n}=67)\end{array}$ & $\begin{array}{l}42.2 \% \\
\quad(\mathrm{n}=49)\end{array}$ & 0.004 & Malpresentation $(\mathrm{n}=97)$ & $\begin{array}{l}47.4 \% \\
\quad(\mathrm{n}=46)\end{array}$ & $\begin{array}{l}52.6 \% \\
\quad(\mathrm{n}=51)\end{array}$ & 0.220 \\
\hline \multicolumn{3}{|l|}{ Time of near-miss occurrence } & 0.069 & \multicolumn{3}{|l|}{ Time of near-miss occurrence } & 0.037 \\
\hline $\begin{array}{l}\text { Near-miss admission } \\
(\mathrm{n}=35)\end{array}$ & $\begin{array}{l}54.3 \% \\
\quad(\mathrm{n}=19)\end{array}$ & $\begin{array}{l}45.7 \% \\
\quad(\mathrm{n}=16)\end{array}$ & & $\begin{array}{l}\text { Near-miss admission } \\
\quad(\mathrm{n}=31)\end{array}$ & $\begin{array}{l}61.3 \% \\
(\mathrm{n}=19)\end{array}$ & $\begin{array}{l}38.7 \%) \\
\quad(\mathrm{n}=12)\end{array}$ & \\
\hline $\begin{array}{l}\text { Near-miss after admission } \\
(\mathrm{n}=32)\end{array}$ & $\begin{array}{l}65.6 \% \\
\quad(\mathrm{n}=21)\end{array}$ & $\begin{array}{l}34.4 \% \\
\quad(\mathrm{n}=11)\end{array}$ & & $\begin{array}{l}\text { Near-miss after admission } \\
\quad(\mathrm{n}=34)\end{array}$ & $\begin{array}{l}50.0 \% \\
\quad(\mathrm{n}=17)\end{array}$ & $\begin{array}{l}50.0 \% \\
\quad(\mathrm{n}=17)\end{array}$ & \\
\hline Time of delivery & & & $<0.001$ & Time of delivery & & & $<0.001$ \\
\hline Afternoon/night $(\mathrm{n}=416)$ & $\begin{array}{l}13.2 \% \\
(\mathrm{n}=55)\end{array}$ & $\begin{array}{l}86.8 \% \\
\quad(\mathrm{n}=361)\end{array}$ & & Afternoon/night $(\mathrm{n}=355)$ & $\begin{array}{l}10.4 \% \\
(\mathrm{n}=37)\end{array}$ & $\begin{array}{l}89.6 \% \\
\quad(\mathrm{n}=318)\end{array}$ & \\
\hline Morning $(\mathrm{n}=344)$ & $\begin{array}{l}61.3 \% \\
\quad(\mathrm{n}=211)\end{array}$ & $\begin{array}{l}38.7 \% \\
\quad(\mathrm{n}=133)\end{array}$ & & Morning $(\mathrm{n}=301)$ & $\begin{array}{l}53.5 \% \\
\quad(\mathrm{n}=161)\end{array}$ & $\begin{array}{l}46.5 \% \\
\quad(\mathrm{n}=140)\end{array}$ & \\
\hline Time of delivery & & & $<0.001$ & Time of delivery & & & $<0.001$ \\
\hline Weekdays $(\mathrm{n}=252)$ & $\begin{array}{l}(38.6 \%) \\
\quad(\mathrm{n}=252)\end{array}$ & $\begin{array}{l}61.4 \% \\
\quad(n=401)\end{array}$ & & Weekdays $(\mathrm{n}=562)$ & $\begin{array}{l}32.9 \% \\
\quad(\mathrm{n}=184)\end{array}$ & $\begin{array}{l}67.1 \% \\
\quad(\mathrm{n}=376)\end{array}$ & \\
\hline Weekend $(n=107)$ & $\begin{array}{l}(13.1 \%) \\
(\mathrm{n}=14)\end{array}$ & $\begin{array}{l}86.9 \% \\
\quad(\mathrm{n}=93)\end{array}$ & & Weekends $(\mathrm{n}=96)$ & $\begin{array}{l}14.6 \% \\
\quad(\mathrm{n}=14)\end{array}$ & $\begin{array}{l}85.4 \% \\
\quad(\mathrm{n}=82)\end{array}$ & \\
\hline
\end{tabular}

${ }^{a}$ Non mutually exclusive indications

associations was quantified by calculating the odds ratio with $95 \% \mathrm{CI}$ and corresponding $p$ values. For predictors of more than two levels, a trend test was also computed using linear representation of the associations in the logistic model. Statistical analyses were carried out with SPSS 17.0 for Windows. 
Table 5 Bivariate analysis of maternal outcome (non-near-miss complication versus near-miss event or death after hospitalization) by diagnosis, comparison of data collected retrospectively and prospectively

\begin{tabular}{|c|c|c|c|c|c|c|c|}
\hline \multicolumn{4}{|c|}{ Retrospective $(\mathrm{N}=1,230)$} & \multicolumn{4}{|c|}{ Prospective $(\mathrm{N}=1,131)$} \\
\hline Final diagnosis & $\begin{array}{l}\text { Complication non } \\
\text { near-miss }(\%) \\
\mathrm{n}=1,061\end{array}$ & $\begin{array}{l}\text { Near-miss or } \\
\text { death }(\%) \\
n=169\end{array}$ & $p$ value & Final diagnosis & $\begin{array}{l}\text { Complication non } \\
\text { near-miss }(\%) \\
\mathrm{n}=954\end{array}$ & $\begin{array}{l}\text { Near-miss or } \\
\text { death }(\%) \\
N=177\end{array}$ & $p$ value \\
\hline $\begin{array}{l}\text { Antepartum } \\
\text { hemorrhage } \\
(\mathrm{n}=85)\end{array}$ & 74.1 & 25.9 & 0.001 & $\begin{array}{l}\text { Antepartum } \\
\text { hemorrhage } \\
(\mathrm{n}=58)\end{array}$ & 75.9 & 24.1 & 0.068 \\
\hline $\begin{array}{l}\text { Postpartum } \\
\text { hemorrhage } \\
(\mathrm{n}=91)\end{array}$ & 53.8 & 46.2 & $<0.001$ & $\begin{array}{l}\text { Postpartum } \\
\text { hemorrhage } \\
(\mathrm{n}=100)\end{array}$ & 50.0 & 50.0 & $<0.001$ \\
\hline $\begin{array}{l}\text { Severe pre/eclampsia } \\
(\mathrm{n}=194)\end{array}$ & 80.4 & 19.6 & 0.010 & $\begin{array}{l}\text { Severe pre/eclampsia } \\
\quad(\mathrm{n}=156)\end{array}$ & 73.1 & 26.9 & $<0.001$ \\
\hline Dystocia (n = 249) & 90.4 & 9.6 & 0.035 & Dystocia $(\mathrm{n}=214)$ & 86.4 & 13.6 & 0.348 \\
\hline $\begin{array}{l}\text { Malpresentation } \\
(\mathrm{n}=137)\end{array}$ & 92.0 & 8.0 & 0.039 & $\begin{array}{l}\text { Malpresentation } \\
(\mathrm{n}=110)\end{array}$ & 91.8 & 8.2 & 0.023 \\
\hline $\begin{array}{r}\text { Miscarriage } \\
(\mathrm{n}=181)\end{array}$ & 93.4 & 6.6 & 0.003 & $\begin{array}{r}\text { Miscarriage } \\
(\mathrm{n}=177)\end{array}$ & 89.8 & 10.2 & 0.029 \\
\hline $\begin{array}{l}\text { Time of near-miss } \\
\text { occurrence }\end{array}$ & & & $<0.001$ & $\begin{array}{l}\text { Time of near-miss } \\
\text { occurrence }\end{array}$ & & & $<0.001$ \\
\hline $\begin{array}{l}\text { Near-miss on } \\
\text { admission }(\mathrm{n}=78)\end{array}$ & 0 & 100 & & $\begin{array}{l}\text { Near-miss on } \\
\text { admission }(\mathrm{n}=85)\end{array}$ & 0 & 100 & \\
\hline $\begin{array}{l}\text { Near-miss after } \\
\text { admission }(\mathrm{n}=53)\end{array}$ & 0 & 100 & & $\begin{array}{l}\text { Near-miss after } \\
\text { admission }(n=62)\end{array}$ & 0 & 100 & \\
\hline $\begin{array}{l}\text { Near-miss unknown } \\
\text { time }(\mathrm{n}=35)\end{array}$ & 0 & 100 & & $\begin{array}{l}\text { Near-miss unclear } \\
(\mathrm{n}=27)\end{array}$ & 0 & 100 & \\
\hline
\end{tabular}

\section{Results}

There were a total of 2,659 obstetric admissions at both sites during the study period, of which 1,404 admissions were from April 1, 2009 to September 31, 2009 (511 women admitted to Kanjuruhan Hospital, and 893 admitted to Bangil Hospital), and 1,255 women from October 1, 2009-May 15, 2010 (533 women admitted to Kanjuruhan Hospital, and 722 admitted to Bangil Hospital). Case notes were found and abstracted on 2,598 (97.7\%) (1,358 retrospective and 1,240 prospective) of these admissions.

Additional data collected through interviews with women and their families were available for $56.8 \%$ of the prospective group $(\mathrm{n}=704)$. Mothers admitted to Bangil Hospital were more educated, $(p=0.03)$, wealthier ( $p=0.021)$, less likely to have been referred directly to the hospital $(p=0.001)$, and had fewer barriers to care $(p=0.011)$ such as geographic, transportation difficulties (data not shown).

There were no differences between those women whose data were collected prospectively or retrospectively with respect to maternal characteristics, type of insurance, place of residence (urban vs. rural), referral patterns, condition on arrival, and time of admission. Significantly more women in the prospective group were $<20$ years old (11.7 vs. $8.6 \%, p=0.012$ ). It is hard to evaluate the relevance between this difference, especially given the inaccurate reporting of birthdates in a rural population with a low literacy level (See Table 2).

Table 3 shows the major reasons for admission to the two hospitals, and illustrates the high-risk nature of these populations; only $9.1 \%(n=237)$ of the deliveries were uncomplicated. There were differences between the data collected during the two periods. Women experiencing other complications (including preterm labor, threatened preterm labor, post-term delivery, polyhydramnios, oligohydramnios, uterine inertia, intrauterine fetal death, premature rupture of membranes, ectopic pregnancy and hyperemesis gravidarum) were encountered more frequently in the prospective group. There was no difference between the two groups either for near-miss events observed on admission $(6.3 \%)$ or which developed during the hospitalization (4.4\%). In this study, most antepartum hemorrhage cases were caused by placenta previa (total and marginal) and low lying placenta (83.2 \%), followed by unknown cause (13.1\%), and placental abruption (3.5\%) (Data not shown). Although this comprised a small proportion, placental abruption was more life threatening compared to placenta previa and unknown antepartum hemorrhage (60 vs. 23.1 (previa) vs. $26.3 \%$ (unknown)). A 
Table 6 Logistical modeling of maternal risk factors associated with maternal near-miss or death after hospitalization

\begin{tabular}{|c|c|c|c|c|c|c|c|c|}
\hline \multirow{2}{*}{$\begin{array}{l}\text { Maternal } \\
\text { characteristics } \\
\text { Demographic }\end{array}$} & \multicolumn{4}{|c|}{ Retrospective-total $(\mathrm{N}=1,358)$} & \multicolumn{4}{|c|}{ Prospective-total $(\mathrm{N}=1,240)$} \\
\hline & $\begin{array}{l}\text { Crude OR } \\
(95 \% \mathrm{CI})\end{array}$ & $p$ value & $\begin{array}{l}\text { Adjusted OR } \\
(95 \% \mathrm{CI})\end{array}$ & $p$ value & $\begin{array}{l}\text { Crude OR } \\
(95 \% \mathrm{CI})\end{array}$ & $p$ value & $\begin{array}{l}\text { Adjusted OR } \\
(95 \% \mathrm{CI})\end{array}$ & $p$ value \\
\hline Multigravidity & $1.54(1.01-2.36)$ & 0.045 & & & $0.68(0.40-1.15)$ & 0.150 & $0.46(0.20-1.08)$ & 0.076 \\
\hline Rural residence & $1.62(1.05-2.52)$ & 0.031 & $1.96(0.94-4.08)$ & 0.072 & $1.63(1.04-2.55)$ & 0.033 & & \\
\hline \multicolumn{9}{|l|}{ Maternal diagnoses } \\
\hline $\begin{array}{l}\text { Antepartum } \\
\text { Hemorrhage }\end{array}$ & $2.68(1.60-4.48)$ & 0.001 & $9.34(4.34-20.13)$ & $<0.001$ & $2.00(1.07-3.74)$ & 0.029 & & \\
\hline Postpartum Hemorrhage & $7.69(4.90-12.08)$ & $<0.001$ & $5.41(2.64-11.08)$ & $<0.001$ & $8.05(5.22-12.42)$ & $<0.001$ & $10.45(5.59-19.52)$ & $<0.001$ \\
\hline $\begin{array}{l}\text { Severe preeclampsia/ } \\
\text { eclampsia }\end{array}$ & $1.92(1.29-2.86)$ & 0.001 & $1.94(1.05-3.57)$ & 0.035 & $2.61(1.76-3.89)$ & $<0.001$ & $3.26(1.79-5.94)$ & $<0.001$ \\
\hline Miscarriage & $0.46(0.25-0.85)$ & 0.013 & & & $0.65(0.39-1.09)$ & 0.100 & $0.037(0.01-0.20)$ & $<0.001$ \\
\hline Other complications & $1.73(1.15-2.60)$ & 0.009 & $1.92(1.04-3.57)$ & 0.038 & $1.54(1.031-2.29)$ & 0.035 & $2.06(1.07-3.95)$ & 0.03 \\
\hline $\begin{array}{l}\text { SES quintile (poorer } \\
\text { vs. richer) }\end{array}$ & NA & NA & NA & NA & $1.77(1.16-2.71)$ & 0.008 & $2.17(1.33-3.54)$ & 0.002 \\
\hline $\begin{array}{l}\text { Place of delivery } \\
\text { (in the hospital } \\
\text { vs. outside) }\end{array}$ & $3.04(2.12-4.38)$ & $<0.001$ & & & $2.93(2.03-4.22)$ & $<0.001$ & $2.04(1.08-3.82)$ & 0.028 \\
\hline
\end{tabular}

Bold values mean that the odds ratios are statistically significant

NA, data not available

large proportion of women had additional diagnoses (data not shown), most frequently being infection, anemia, premature rupture of membranes and premature labor.

Table 4 displays the mode of delivery by principal diagnosis in the retrospective and prospective data sets. The diagnoses associated with significantly higher rates $(p<0.001)$ of cesarean delivery compared to vaginal delivery in both data sets included antepartum hemorrhage and dystocia In both data sets, significantly more cesarean sections occurred during the morning shift compared to afternoon or evenings $(p<0.001)$ and on weekdays compared to weekends $(p<0.001)$.

Table 5 describes the bivariate analyses for maternal outcomes for each major diagnostic category. Mothers with no complications were excluded from this analysis. In both the retrospective and prospective data, women with diagnoses of postpartum hemorrhage or severe preeclampsia/ eclampsia were significantly different for women with non near-miss and women with near-miss or death. Table 6 shows crude and adjusted risks of adverse maternal outcome (near-miss or death) for both retrospective and prospective data. Among the diagnoses, postpartum hemorrhage (AOR 5.41, $95 \%$ CI 2.64-11.08, retrospective; AOR 10.45, $95 \%$ CI 5.59-19.52, prospective) and severe pre-eclampsia/eclampsia (AOR 1.94, $95 \%$ CI 1.05-3.57, retrospective; AOR 3.26, $95 \%$ CI 1.79-5.94, prospective) were found to be consistently associated with an increased risk of near-miss or death after adjustment for other variables (maternal age, gravidity, education, employment, referral from other health facility, time of admission and delivery, insurance, maternal diagnoses, barriers to care, place of delivery). The risk ratio for prospective compared to the retrospective data was 1.93 for postpartum hemorrhage and was 1.68 for severe preeclampsia/eclampsia.

Six mothers died. Maternal deaths were due to antepartum hemorrhage $(\mathrm{n}=1)$, postpartum hemorrhage $(\mathrm{n}=1)$, severe preeclampsia/eclampsia $(\mathrm{n}=2)$, and amniotic fluid embolism $(n=1)$. The cause of one death is unknown as the case notes could not be located.

\section{Conclusions}

Maternal death and morbidity in Indonesia have declined over the last decade with enhanced training of providers. Persistent delays in seeking care, geographic and transportation barriers persist. Such factors singularly or combined may explain the majority of deaths and morbidity occurring at the community level. $44 \%$ of maternal deaths occur in either district or provincial hospitals $[9,30]$. Such deaths could be preventable if optimal hospital care were timely and accessible. Understanding the maternal characteristics and diagnoses associated with the worst outcomes may help hospitals identify needed service improvements.

This study was part of an effort to assist the Ministry of Health in improving obstetrical services offered within the public health delivery system. The results of this study should direct the supplemental training at the district 
hospital levels and focus on physicians and nursing staff as well as auxiliary health professions. To improve care, the emergency transportation system needs both development and investment.

Rates of near-miss or death amongst mothers in this study are alarming. As previously noted, a systematic review on the prevalence of near-miss events, based on disease criteria, ranged between 0.6 and $14.98 \%$ [13]. More than $13 \%$ of women in our study were categorized as near-miss either on admission or during hospitalization, more than triple the rate reported from Brazil [31]. The ratio of mortality to near miss in our study was $1: 25$, lower than reported in another Indonesian study [25]. A significant proportion of our cases reached near-miss status after admission, suggesting that improvements in hospital practices/response time (especially triage and emergency obstetric care) could help improve outcomes.

Globally, the leading causes of maternal morbidity and mortality are hypertensive disorders of pregnancy and hemorrhage [13]. As in our study, postpartum hemorrhage was previously reported as the leading cause of maternal death in Indonesia [8, 32], followed by antepartum hemorrhage, severe preeclampsia and eclampsia. Of the women with postpartum hemorrhage, $50 \%$ may reach near-miss status. A major cause of postpartum hemorrhage among our women was retained products of conception, suggesting the possibility that management of the third stage of labor at the community level is suboptimal. Other developing countries have reduced rates of hemorrhage by the appropriate use of misoprostol; a drug that is inexpensive and does not require refrigeration [33, 34]. The low rate (7.4\%) of postpartum hemorrhage in our women raises the possibility that many women with this complication may be dying in the community rather than in the hospital. The majority of women were from rural areas where transportation difficulties also contribute to the poor outcomes; these data support the need for broader availability of misoprostol or other oxytocic agents to prevent and treat postpartum hemorrhage. The results of our study show a lower incidence of antepartum compared to postpartum hemorrhage among hospital admissions. Its contribution to near-miss status is as high as postpartum hemorrhage among women delivering at the hospital. Women who deliver prior to hospitalization are not likely to progress to a near-miss status once hospitalized. It is difficult to estimate what percentage of these mothers may have died in the community. Almost $14 \%$ of women had severe preeclampsia and eclampsia, half of those delivering by cesarean. Twenty to twenty-seven percent of these cases progressed to near-miss status or death, which is higher than in similar populations [30]. Possible explanations include high levels of acuity at presentation, delays in or mismanaged care. Early diagnosis of hypertension during pregnancy and appropriate referral may help improve outcomes. Easy access and appropriate use of magnesium sulfate when indicated before and during transport and upon admission may further reduce morbidity.

The most frequent diagnosis associated with hospital admission was dystocia, with more than one-third delivering vaginally. We hypothesize that improved skills in the community could have prevented some of these referrals. Although the rates of near-miss were less frequent for women with dystocia when compared to other diagnoses, they exceeded those associated with antepartum hemorrhage. Improved training for midwives and obstetricians could reduce this category of near-miss significantly.

Due to the spectrum of diagnoses associated with antepartum hemorrhage there may not be a single strategy to help mitigate its impact on maternal morbidity and mortality. The value of prenatal care in the community health care setting in diagnosing cases at increased risk for antepartum hemorrhage cannot be overestimated. Comparison to the published literature is difficult as a recent systematic review and meta-analysis of maternal hemorrhage could find no studies with consistent definitions [35]. Our findings should be understood in light of the strengths and limitations. The sample is poverty-stricken, as evidenced by the fact that almost half received government insurance for the poor. Not all pregnant women who qualified for a poverty letter were able to procure one. Frequently, a letter was sought during an emergency requiring hospitalization; often prompted by the hospital officials requesting this letter. This represents a limitation in our data since pregnant women with limited economic resources and serious medical complications may be misclassified in our study or not included. In general women who did not seek care at a government hospital regardless of economic status are not included in this study. Therefore, the results may not be generalizable to the total population. A strength of the study is that we likely captured most of the near-miss cases and deaths at the hospital level since severe cases in Indonesia are usually referred to a government hospital. While we did manage to review and abstract medical records for almost $99 \%$ of prospective patients, interviews were available for only $56.8 \%$ of these women. Under coverage bias may exist for the data collected via interview, such as maternal education, maternal employments status, referral chain, length of time and barriers to seeking care. These variables provide a qualitative look at the lives of these women and as such, do enrich the data. It is possible that if we had more complete coverage, these variables might have reached significance in the logistic regression.

This comprehensive review provided insight into areas that could potentially reduce obstetric mortality and morbidity in Indonesia. Understanding the common diagnoses 
and the quality of care delivered should inform policy makers about potential solutions. Creation of standard protocols and structured provider education may be beneficial. At the community level, prompt recognition of complications and timely referral of more intensive care should be helpful. Prompt triage in the emergency rooms and labor wards is essential as well. The lack of trained personnel and limited numbers of obstetricians in district hospitals is a barrier. Collaboration between emergency room physicians, midwives and labor ward obstetricians could allow for prompt treatment of pregnant and recently pregnant women. The adjusted odds ratios for near-miss or death due to postpartum hemorrhage and/or severe preeclampsia/eclampsia suggest that the factors related to hospital readiness in providing care for emergency obstetric cases are substantially more important than factors from the community side (barriers and access to care). This is supported by our results that found no significant increased risk due to personal and access barriers, either as an individual variable or combined (data not shown). Earlier institution of life-saving care would be of great value. Indonesia has invested heavily in creating a solid primary care structure, with less investment in secondary and tertiary levels of care. This study demonstrates that despite the investment in a strong primary health service network, the results are similar to those reported in developing economies with less intensive primary systems. The conclusion to be drawn is that without a more ambitious investment in secondary and tertiary care, incidence of these maternal complications and the associated morbidity may not be easily improved. These conclusions match those of Souza et al. [36] who determined that in order to reduce maternal mortality, there is a substantial need to improve comprehensive emergency care.

Collecting both retrospective and prospective data allowed us to assess the quality of the data collected retrospectively. Prospectively collected medical data provided an opportunity to investigate important non-clinical information. Otherwise, it was comparable to the retrospective data. The importance of our findings confirming no difference in the results of the analyses of prospectively and retrospectively collected data is of particular importance to future researchers. Retrospectively collected data may be more readily available through existing medical records and to our satisfaction reaches an equivalent level of accuracy to prospectively collected information. None-theless the data obtained from interviewing women and their families are invaluable in better understanding barriers to care and referral patterns. These included visits to multiple sites within the health care system prior to arriving at the district hospital. Some differences noted are intriguing and must be left to interpretation. For example, the association between multigravidity and near-miss status was significant only in the retrospectively collected data. Further studies to better understand how to overcome barriers to preventing near miss and death are essential. Our analyses are one step in the right direction.

Acknowledgments This study is made possible by the generous support of the American people through the United States Agency for International Development (USAID). The contents are the responsibility of John Snow, Inc. and do not necessarily reflect the views of USAID or the United States Government. This research was also supported [in part] by the Intramural Research Program of the NIH, Eunice Kennedy Shriver National Institute of Child Health and Human Development.

\section{References}

1. World Health Organization (WHO). (2014). Trends in maternal mortality: 1990 to 2013. Estimates by WHO, UNICEF, UNFPA, the World Bank and the United Nations Population Division. Geneva, World Health Organization. http://apps.who.int/iris/bit stream/10665/112682/2/9789241507226_eng.pdf?ua=1. Retrieved November 11, 2014.

2. World Health Organization (WHO). (2014). Maternal mortality Fact sheet No. 348, Geneva, World Health Organization. http://www. who.int/mediacentre/factsheets/fs348/en/. Retrieved November 7, 2014.

3. Hill, K., Abou Zhar, C., \& Wardlaw, T. (2001). Estimates of maternal mortality for 1995. Bulletin of the World Health Organization, 79, 182-193.

4. Hogan, M. C., Foreman, K. J., Naghavi, M., Ahn, S. Y., Wang, M., Makela, S. M., et al. (2010). Maternal mortality for 181 countries, 1980-2008: a systematic analysis of progress towards Millennium Development Goal. Lancet,. doi:10.1016/S01406736(10)60518-1.

5. Statistics Indonesia (Badan Pusat Statistik-BPS), National Population and Family Planning Board (BKKBN), and Kementerian Kesehatan (Kemenkes-MOH), and ICF International. (2013). Indonesia Demographic and Health Survey 2012. Jakarta: BPS, BKKBN, Kemenkes, and ICF International.

6. WHO and UNICEF. (2013). Accountability for maternal, newborn and child survival: The 2013 update. Geneva. http://www. countdown2015mnch.org/documents/2013Report/Countdown 2013-Update_withprofiles.pdf. Retrieved November 17, 2014.

7. Ronsmans, C., Scott, S., Qomariyah, S. N., Achadi, E., Braunholtz, D., Marshall, T., et al. (2009). Professional assistance during birth and maternal mortality in two Indonesian districts. Bulletin of the World Health Organization, 87, 416-423.

8. Hermiyanti S. The challenge of making safe motherhood a reality: Community midwives in Indonesia. 2008. Ahmedabad. http://209.61.208.233/LinkFiles/FCH_d3-7challenges-msm-ino. pdf. Retrieved December 10, 2013.

9. Ronsmans, C, \& Filippi, V. (2004). Reviewing severe maternal morbidity: learning from women who survive life threatening complications. In: G. Lewis (Ed.), Beyond the numbers. Reviewing maternal deaths and complications to make pregnancy safer, pp. 103-123. Geneva: World Health Organization. http:// whqlibdoc.who.int/publications/2004/9241591838.pdf. Retrieved December 2, 2013.

10. Badan Pusat Statistik-Statistics Indonesia (BPS) [Indonesia], ORC Macro. (2007). Indonesia demographic and health survey 2007. Calverton, Maryland: BPS and ORC Macro. http://www. measuredhs.com/pubs/pdf/FR218/FR218[27August2010].pdf. Retrieved December 17, 2013. 
11. Supratiko, G., Wirth, M. E., Achadi, E., Cohen, S., \& Ronsman, S. C. (2002). A district-based audit of the causes and circumstances of maternal deaths in South Kalimantan, Indonesia. Bulletin of the World Health Organization, 80, 228-234.

12. Ministry of Health [Indonesia]. (2001). National strategic plan for making pregnancy safer (MPS) in Indonesia 2001-2010. Jakarta: Ministry of Health.

13. Tuncalp, O., Hindin, M. J., Souza, J. P., Chou, D., \& Say, L. (2012). The prevalence of maternal near miss: A systematic review. BJOG, 119, 653-661.

14. Oladapo, O. T., Sule-Odu, A. O., Olatunji, A. O., \& Daniel, O. J. (2005). "Near-miss" obstetric events and maternal deaths in Sagamu, Nigeria: A retrospective study. Reproductive Health, 2, 9. doi:10.1186/1742-4755-2-9.

15. Mustafa, R., \& Hashmi, H. (2009). Near-miss obstetrical events and maternal deaths. Journal of the College of Physicians and Surgeons Pakistan, 19, 781-785.

16. Shrestha, N. S., Saha, R., \& Karki, C. (2010). Near miss maternal morbidity and maternal mortality at Kathmandu Medical College Teaching Hospital. Kathmandu University Medical Journal, 8, 222-226.

17. Lotufo, F. A., Parpinelli, M. A., Haddad, S. M., Surita, F. G., \& Cecatti, J. G. (2012). Applying the new concept of maternal nearmiss in an intensive care unit. Clinics (Sao Paulo), 67, 225-230.

18. Souza, J. P., Cecatti, J. G., Faundes, A., Morais, S. S., Villar, J., Carroli, G., et al. (2010). Maternal near miss and maternal death in the World Health Organization's 2005 global survey on maternal and perinatal health. Bulletin of the World Health Organization, 88, 113-119.

19. Waterstone, M., Bewley, S., \& Wolfe, C. (2001). Incidence and predictors of severe obstetric morbidity: Case control study. BMJ, 322, 1089-1094.

20. World Health Organization. (2014). Global health observatory maternal mortality country profiles. http://www.who.int/gho/ maternal_health/countries/en/. Retrieved November 24, 2014.

21. WHO. (2011). Evaluating the quality of care for severe pregnancy complications: The WHO near-miss approach for maternal health. http://whqlibdoc.who.int/publications/2011/9789241502221_eng. pdf. Retrieved January 9, 2014.

22. Pattinson, R. C., \& Hall, M. (2003). Near-misses: A useful adjunct to maternal death enquiries. British Medical Bulletin, 67, 231-243.

23. Haddad, S. M., Cecatti, J. G., Souza, J. P., Sousa, M. H., Parpinelli, M. A., Costa, M. L., et al. (2014). Applying the maternal near miss miss approach for the evaluation of quality of obstetric care: A worked example from a Multicenter Surveillance Study. BioMed Research International. doi:10.1155/2014/989815.

24. Say, L., Souza, J. P., \& Pattinson, R. C. (2009). Maternal near miss: towards a standard tool for monitoring quality of maternal health care. Best Practice and Research: Clinical Obstetrics and Gynaecology, 23, 287-296.
25. Adisasmita, A., Deviany, P. E., Nandiaty, F., Stanton, C., \& Ronsmans, C. (2008). Obstetric near miss and deaths in public and private hospitals in Indonesia. BMC Pregnancy Childbirth, 8, 10.

26. Mantel, G. D., Buchmann, E., Rees, H., \& Pattinson, R. C. (1998). Severe acute maternal morbidity: A pilot study of a definition of a near miss. BJOG, 105, 985-990.

27. Oladapo, O. T., Adetoro, O. O., Fakeye, O., Ekele, B. A., Fawole, A. O., Abasiattai, A., et al. (2009). Nigerian Network for Reproductive Health Research and Training (NNRHRT). National data system on near miss and maternal death: shifting from maternal risk to public health impact in Nigeria. Reproductive Health, 6,8 .

28. Central Bureau of Statistics (CBS) [Indonesia], State Ministry of Population/National Family Planning Coordinating Board, Ministry of Health, and Macro International. (1998). Indonesia demographic and health survey 1997. Calverton, Maryland: CBS and Macro International. http://pdf.usaid.gov/pdf_docs/PNACD956. pdf. Retrieved December 4, 2013.

29. Hosmer, D. W., \& Lemeshow, S. (2000). Applied logistic regression (2nd ed.). New York: Wiley.

30. Ministry of Health. (2001). Indonesia national household health survey (Surkesnas). Mortality Study. National Institute of Health Research and Development (NIHRD): Jakarta.

31. Amaral, E., Souza, J. P., Surita, F., Luz, A. G., Sousa, M. H., Cecatti, J. G., et al. (2011). A population-based surveillance study on severe acute maternal morbidity (near-miss) and adverse perinatal outcomes in Campinas, Brazil: The Vigimoma Project. BMC Pregnancy Childbirth, 11, 9.

32. Supratikto, G., Wirth, M. E., Achadi, E., Cohen, S., \& Ronsmans, C. (2002). A district-based audit of the causes and circumstances of maternal deaths in south Kalimantan,Indonesia. Bulletin of the World Health Organization, 80, 228-235.

33. Blum, J., Winikoff, B., Raghavan, S., Dabash, R., Ramadan, M. C., Dilbaz, B, et al. (2010). Treatment of post-partum haemorrhage with sublingual misoprostol versus oxytocin in women receiving prophylactic oxytocin: a double blind, randomized noninferiority trial. Lancet 375-176-8.

34. Winokoff, B., Dabash, R., Durocher, J., Darwish, E., Nguyen, T. N., León, W., et al. (2010). Treatment of postpartum hemorrhage with misoprostol versus oxytocin in women not exposed to oxytocin during labour: A double-blind, randomized, non-inferiority trial. Lancet, 375, 210-216.

35. Calvert, C., Thomas, S. L., Ronsmans, C., Wagner, K., Adler, A. J., \& Filippi, V. (2012). Identifying regional variation in the prevalence of postpartum haemorrhage: A systematic review and meta-analysis. PLoS One, 7(7), e41114. doi:10.1371/journal. pone.0041114.

36. Souza, J. P., Gülmezoglu, A. M., Vogel, J., et al. (2013). Moving beyond essential interventions for reduction of maternal mortality (the WHO Multicountry Survey on Maternal and Newborn Health): A cross-sectional study. Lancet, 381, 1747-1755. 